\title{
SOME ASYMPTOTIC FORMULAS IN THE THEORY OF NUMBERS $\left({ }^{1}\right)$
}

BY

ECKFORD COHEN

1. Introduction. It is the aim of this paper to generalize, unify, and refine a number of related but scattered results in asymptotic number theory. Special cases of the main theorems of the paper yield estimates due to Cesàro [2], Feller and Tornier [10], Kanold [12], Rényi [13], and the present author [4]. The method of proof is elementary and is essentially a variant of a method used in an earlier note [7].

Clearly, every positive integer $n$ has a unique representation of the form

$$
n=d^{2} e, \quad e \text { square-free, } d>0 .
$$

If in this relation we place $d=Q(n)$, then $Q^{2}(n)$ is the largest square divisor of $n$. Let $\alpha$ denote an arbitrary non-negative real number and let $S$ be any nonvacuous subset of the set $J$ of positive integers. In Theorem 2.1 we obtain approximations to the sum

$$
B_{\alpha}(x, S) \equiv \sum_{n \leqq x \cdot Q(n) \in S}\left(\frac{n}{Q^{2}(n)}\right)^{\alpha}, \quad x \geqq 1,
$$

with numerous special results deduced as corollaries.

We shall say that a divisor $d$ of $n$ is unitary, written $d \| n$ or $d * \delta=n$, if $d \delta=n$, $(d, \delta)=1$. In $\$ 3$ we consider the sum $B_{\alpha}^{\prime}(x, S)$ arising from (1.2) if, in the summation, $Q^{2}(n)$ is required to be a unitary divisor of $n$. The main result is contained in Theorem 3.1.

Let the distinct prime factors of $n$ be denoted $p_{1}, \cdots, p_{r}$, and place

$$
n=p_{1}^{e_{1}} \cdots p_{r}^{e_{r}} \quad(r=0 \text { if } n=1) .
$$

The set of integers $n$ for which $e_{i} \geqq 2(i=1, \cdot \cdot, r)$, that is, the integers with no simple prime factors, will be denoted by $L$. Evidently, $n$ is uniquely representable in the form

$$
n=d e, \quad d \in L, e \text { square-free, }(d, e)=1 .
$$

The factor $d$ defined by (1.4) is the largest divisor $Q^{*}(n)$ of $n$ contained in $L$. If $T$ is a nonvacuous subset of $L$, then one may define, analogous to (1.2),

Presented to the Society, March 29, 1961 ; received by the editors August 21, 1962.

(1) This research was supported in part by the National Science Foundation (G-19012). 


$$
B_{\alpha}^{*}(x, T)=\sum_{n \leqq x ; Q^{*}(n) \in T}\left(\frac{n}{Q^{*}(n)}\right)^{\alpha} .
$$

An estimate for $B_{\alpha}^{*}(x, T)$ is proved in Theorem 4.1.

Known results which are deduced as special cases of the theorems discussed above are listed as follows: Corollaries 2.1.2., 2.3.2, 2.4.2, 2.7.2, 2.8.2, 3.2.4, 4.1.1, 4.2.1, 4.2.2. It will be observed that in the case $\alpha=0$, the sums $B_{\alpha}(x, S)$, $B_{\alpha}^{\prime}(x, S)$, and $B_{\alpha}^{*}(x, T)$ become enumerative functions of certain sequences of integers. This special case is given particular emphasis in each of the three problems considered.

We confine our description of specific results of the paper to a single type that has recently stimulated interest $[1 ; 11 ; 13 ; 16]$. Referring to $(1.3)$, let us place $\omega(n)=r$ and $\Omega(n)=e_{1}+\cdots+e_{r}$. Further, place

$$
\Delta(n)=\Omega(n)-\omega(n)=\Delta\left(Q^{*}(n)\right) .
$$

Rényi has proved $[13,(3)]$ that if $d_{m}^{*}$ is the asymptotic density of the sequence of integers $n$ for which $\Delta(n)=m$, then $d_{m}^{*}$ exists for each integer $m \geqq 0$, and

$$
\sum_{m=0}^{\infty} d_{m}^{*} z^{m}=\prod_{p}\left(1-\frac{1}{p}\right)\left(1+\frac{1}{p-z}\right), \quad|z|<2,
$$

where the product is extended over the primes $p$. A new proof of this result is given in $\S 4$.

We also prove two analogues of (1.7). In particular, let $d_{m}$ denote the density of the set of integers $n$ such that $\Delta\left(Q^{2}(n)\right)=m$; then, by $\S 2$, each $d_{m}$ exists and

$$
\sum_{m=0}^{\infty} d_{m} z^{m}=\prod_{p}\left(1-\frac{1}{p^{2}}\right)\left(1+\frac{z}{p^{2}-z^{2}}\right), \quad|z|<2 .
$$

Moreover, it is shown (§3) that if $d_{m}^{\prime}$ represents the density of the set of $n$ for which $\Delta\left(Q^{2}(n)\right)=m$, where $Q^{2}(n) \| n$, then

$$
\sum_{m=0}^{\infty} d_{m}^{\prime} z^{m}=\prod_{p}\left(1-\frac{1}{p}\right)\left(1+\frac{1}{p}+\frac{z}{p^{2}-z^{2}}\right), \quad|z|<2 .
$$

Remark 1.1. With $z=1$ in (1.8) we note that

$$
\sum_{m=0}^{\infty} d_{m}=1
$$

by (1.7) the corresponding result for $d_{m}^{*}$ is also valid (Rényi [13]).

2. Problem I. Estimates for $B_{\alpha}(x, S)$. Let $\Gamma_{\alpha}(n, S)$ be defined by

$$
\Gamma_{\alpha}(n, S)=\left\{\begin{array}{cc}
\left(\frac{n}{Q^{2}(n)}\right)^{\alpha} & \text { if } Q(n) \in S, \\
0 & \text { if } Q(n) \notin S .
\end{array}\right.
$$


In particular, $\Gamma_{0}(n, S)$ is the characteristic function of the set of integers such that $Q(n) \in S$.

With $\mu(n)$ denoting the Möbius function, we have the following relation which is basic for this section.

LEMMA 2.1.

$$
\Gamma_{\alpha}(n, S)=\sum_{d^{2} e=n ; d \in S} e^{\alpha} \mu^{2}(e)
$$

This formula is merely a restatement of the uniqueness of the representation (1.1). The following known estimate is also required.

LEMMA 2.2 [4, LeMma $4.2, r=1]$. If $s \geqq 0$, then for $x \geqq 1$,

$$
\mho_{s}(x) \equiv \sum_{n \leqq x} n^{s} \mu^{2}(n)=\frac{6}{\pi^{2}}\left(\frac{x^{s+1}}{s+1}\right)+O\left(x^{s+1 / 2}\right) .
$$

Define $\zeta_{s}(s)$ to be the sum of the series,

$$
\zeta_{S}(s) \equiv \sum_{n=1 ; n \in S}^{\infty} \frac{1}{n^{s}}
$$

for those sequences $S$ and real numbers $s$ for which the series converges. Note that $\zeta_{J}(s)$ is the zeta-function $\zeta(s)$, provided $s>1$.

We are now ready to prove the main theorem of this section.

THEOREM 2.1. If $\alpha \geqq 0$, then for $x \geqq 2$,

$$
B_{\alpha}(x, S)=\frac{6}{\pi^{2}}\left(\frac{x^{\alpha+1}}{\alpha+1}\right) \zeta_{s}(2 \alpha+2)+O\left(\left(x^{\alpha+1 / 2} R_{\alpha}(x, S)\right),\right.
$$

uniformly in $S$, where

$$
R_{\alpha}(x, S)=\sum_{n \leqq \sqrt{ } x ; n \in S} n^{-2 \alpha-1}
$$

provided this sum is nonvacuous; otherwise $R_{\alpha}(x, S)=1$.

REMARK 2.1. Evidently, $R_{0}(x, S)=O(1)$ for those $S$ for which $\zeta_{S}(1)$ is finite. Moreover, for arbitrary $S$,

$$
R_{\alpha}(x, S)= \begin{cases}O(1) & \text { if } \alpha>0 \\ O(\log x) & \text { if } \alpha=0\end{cases}
$$

In particular, the estimate, $R_{\alpha}(x, S)=O(\log x)$, is always valid.

Proof. By (1.2), (2.1), (2.3), and Lemma 2.1,

$$
B_{\alpha}(x, S)=\sum_{n \leqq x} \Gamma_{\alpha}(n, S)=\sum_{d^{2} e \leqq x ; d \in S} e^{\alpha} \mu^{2}(e)=\sum_{n \leqq \sqrt{ } ; n \in S} \mho_{\alpha}\left(\frac{x}{n^{2}}\right) .
$$


Then by Lemma 2.2

$$
\begin{aligned}
B_{\alpha}(x, S) & =\frac{6}{\pi^{2}}\left(\frac{x^{\alpha+1}}{\alpha+1}\right) \sum_{n \leqq \downarrow x ; n \in S} n^{-2 \alpha-2}+O\left(x^{\alpha+1 / 2} R_{\alpha}(x, S)\right) \\
& =\frac{6}{\pi^{2}}\left(\frac{x^{\alpha+1}}{\alpha+1}\right) \zeta_{S}(2 \alpha+2)+O\left(x^{\alpha+1} \sum_{n>\sqrt{ } x} n^{-2 \alpha-2}\right)+O\left(x^{\alpha+1 / 2} R_{\alpha}(x, S)\right) .
\end{aligned}
$$

But the summation in the middle term is $O\left(x^{-\alpha-1 / 2}\right)$ and therefore (2.5) results.

We now specialize the theorem to some special sets of integers $S$. Let $H$ denote the set of integral squares.

COROLlaRY 2.1. If $\alpha \geqq 0$, then

$$
B_{\alpha}(x, H)=\frac{6}{\pi^{2}}\left(\frac{x^{\alpha+1}}{\alpha+1}\right) \zeta(4 \alpha+4)+O\left(x^{\alpha+1 / 2}\right) \text {. }
$$

Place $B_{0}(x, S)=B(x, S)$ for all $S$. Since $\zeta(4)=\pi^{4} / 90$, one obtains

Corollary 2.1.1 $(\alpha=0)$.

$$
B(x, H)=\frac{\pi^{2} x}{15}+O(\sqrt{ } x)
$$

COROLLARY 2.1.2 (CESÁRO $[2, \S 17])$. The asymptotic density of the integers $n$ for which $Q(n)$ is a square is $\pi^{2} / 15$.

Suppose that $S_{d}$ is the set of all $n$ divisible by the fixed positive integer $d$.

COROLlaRY 2.2. If $\alpha>0$, then

$$
B_{\alpha}\left(x, S_{d}\right)=\frac{6}{\pi^{2}}\left(\frac{x^{\alpha+1}}{\alpha+1}\right) \frac{\zeta(2 \alpha+2)}{d^{2 \alpha+2}}+O\left(x^{\alpha+1 / 2}\right) .
$$

Noting that $S_{1}=J$, we have

Corollary 2.2.1 $(d=1)$. If $\alpha>0$, then

$$
B_{\alpha}(x, J)=\frac{6}{\pi^{2}}\left(\frac{x^{\alpha+1}}{\alpha+1}\right) \zeta(2 \alpha+2)+O\left(x^{\alpha+1 / 2}\right) .
$$

We say that an arithmetical function $f(n)$ has average order $E(x)$ if its summatory function $F(x) \sim x E(x)$ as $x \rightarrow \infty$. Note that $B_{\alpha}(x, J)$ is the summatory function of $\Gamma_{\alpha}(n, J)$.

COROLlaRY 2.2.2. If $\alpha>0$, then the $\alpha$ th power of the divisor of $n$ conjugate to the greatest square divisor of $n$ has average order $\left(6 x^{\alpha} / \pi^{2}\right)(\zeta(2 \alpha+2) /(\alpha+1))$.

COROLlaRY 2.2.3 $(d=\alpha=1)$. The conjugate divisor of the greatest square divisor of $n$ has average order $\pi^{2} x / 30$.

Next we consider the case in which $S$ consists of a single positive integer $r, S \equiv r$. 
COROllary 2.2. For all $\alpha \geqq 0$,

$$
B_{\alpha}(x, r)=\frac{6}{\pi^{2}}\left(\frac{x^{\alpha+1}}{\alpha+1}\right) r^{-2 \alpha-2}+O\left(\frac{x^{\alpha+1 / 2}}{r^{2 \alpha+1}}\right)
$$

uniformly in $r \leqq \sqrt{ } x$.

Corollary 2.3.1 $(\alpha=0)$.

$$
B(x, r)=\frac{6 x}{\pi^{2} r^{2}}+O(\sqrt{ } x) .
$$

Corollary 2.3.2 (KANOld [12, (13)]; ALSO CF. SCHERK $[14,(3)])$. The asymptotic density of the set of integers $n$ with $Q(n)>r$ is $1-\left(6 / \pi^{2}\right) \Sigma_{a \leqq r} a^{-2}$.

Let $D$ denote the sequence of square-free integers. Since the generating function of $D, \sum_{n=1}^{\infty} \mu^{2}(n) / n^{s}=\zeta(s) / \zeta(2 s), s>1$, we have

COROLlary 2.4. For all $\alpha \geqq 0, x \geqq 2$,

$$
B_{\alpha}(x, D)=\frac{6}{\pi^{2}}\left(\frac{x^{\alpha+1}}{\alpha+1}\right) \frac{\zeta(2 \alpha+2)}{\zeta(4 \alpha+4)}+ \begin{cases}O\left(x^{\alpha+1 / 2}\right) & \text { if } \alpha>0, \\ O(\sqrt{ } x \log x) & \text { if } \alpha=0 .\end{cases}
$$

Corollary 2.4.1 $(\alpha=0)$. If $x \geqq 2$, then

$$
B(x, D)=\frac{90 x}{\pi^{4}}+O(\sqrt{ } x \log x) \text {. }
$$

Corollary 2.4.2 (CESÀro[2, $\$ 21]$ ). The asymptotic density of the set of those $n$ for which $Q(n)$ is square-free is $90 / \pi^{4}$.

Let $P$ denote the set of the primes. Since

$$
\sum_{p \leqq x} \frac{1}{p}=O(\log \log x), \quad x \geqq 3,
$$

we have

COROLlary 2.5. If $x \geqq 3$, then

$$
B_{\alpha}(x, P)=\frac{6}{\pi^{2}}\left(\frac{x^{\alpha+1}}{\alpha+1}\right) \sum_{p} \frac{1}{p^{2 \alpha+2}}+ \begin{cases}O\left(x^{1 / 2+\alpha}\right) & \text { if } \alpha>0 \\ O(\sqrt{ } x \log \log x) & \text { if } \alpha=0\end{cases}
$$

COROLlaRY 2.5.1 $(\alpha=0)$. The asymptotic density of the integers for which $Q(n)$ is prime is equal to

$$
\frac{6}{\pi^{2}} \sum_{p} \frac{1}{p^{2}}
$$

Let $\left\{a_{1}, \cdots, a_{s}\right\}$ denote an ordered set of $s$ fixed positive integers, $s \geqq 0$, and let $N=N_{s}(a)$ denote the set of all $n$ of the form $n=p_{1}^{a_{1}} \cdots p_{s}^{a_{s}}$, where the $p_{i}$ are 
distinct primes. In case $s=0$, it is understood that $N=(1)$. Then by (2.17) and the above theorem,

COROLlaRY 2.6. If $x \geqq 3$,

$$
B_{\alpha}(x, N)=\frac{6}{\pi^{2}}\left(\frac{x^{\alpha+1}}{\alpha+1}\right) \zeta_{N}(2 \alpha+2)+ \begin{cases}O\left(x^{\alpha+1 / 2}\right) & \text { if } \alpha>0 \\ O\left(\sqrt{x}(\log \log x)^{t}\right) & \text { if } \alpha=0\end{cases}
$$

where $t=t(a)$ denotes the number of 1 's in the set, $a_{1}, \cdots, a_{s}$.

COROllary 2.6.1 $(\alpha=0)$. The asymptotic density of the integers $n$ for which $Q(n) \in N_{s}(a)$ is given by

$$
\delta_{s}(a)=\frac{6}{\pi^{2}} \sum_{p_{1}, \ldots, p_{s}} \frac{1}{\left(p_{1}^{a} \cdots p_{s}^{a}\right)^{2}}
$$

where the summation is over all s-tuples of distinct primes, $p_{1}, \cdots, p_{s}$, for which the numbers, $p_{1}^{a_{1}} \cdots p_{s}^{a_{s}}$ are distinct; if $s=0$, the sum is understood to have the value 1.

Note that Corollary 2.6.1 reduces to Corollary 2.5.1 in case $s=1, a_{1}=1$. We now prove (1.8) as a consequence of (2.20).

COROllaRY 2.6.2. If $d_{m}$ is defined as in the introduction, then for $|z|<2$,

$$
\sum_{m=0}^{\infty} d_{m} z^{m}=\frac{6}{\pi^{2}} \prod_{p}\left(1+\frac{z}{p^{2}-z^{2}}\right) .
$$

Proof. By definition of $d_{m}$ and (2.20)

$$
d_{m}=\frac{6}{\pi^{2}} \sum_{p_{1}, \ldots, p_{s} ; s \geqq 0} \sum_{a_{1}, \ldots, a_{s}} \frac{1}{\left(p_{1}^{a} \cdots p_{s}^{a}\right)^{2}},
$$

where for each set of $s$ distinct primes, $p_{1}, \cdots, p_{s}$, the numbers $a_{1}, \cdots, a_{s}$ range over all $s$-tuples of positive integers such that $\left(2 a_{1}-1\right)+\cdots+\left(2 a_{s}-1\right)=m$. Equivalently,

$$
d_{m}=\frac{6}{\pi^{2}} \sum_{p_{1}, \ldots, p_{s} ; s \geqq 0} \frac{1}{p_{1} \cdots p_{s}} \sum_{b_{1}, \ldots, b_{s} ; b \text { odd }} \frac{1}{p_{1}^{b_{1} \cdots p_{s}^{b_{s}}},}
$$

where the numbers $b_{1}, \cdots, b_{s}$, for each $s \geqq 0$, range over all $s$-tuples of odd positive integers such that $b_{1}+\cdots+b_{s}=m$. It then follows that

$$
\sum_{m=0}^{\infty} d_{m} z^{m}=\frac{6}{\pi^{2}} \prod_{p}\left(1+\frac{1}{p}\left(\frac{z}{p}+\frac{z^{3}}{p^{3}}+\frac{z^{5}}{p^{5}}+\cdots\right)\right),
$$

from which (2.21) results.

The formula (1.8) follows from (2.21) by virtue of the relation $\zeta(2)=6 / \pi^{2}$ and the Euler product representation of $\zeta(s)$. 
Let $E$ denote the set of all $n$ such that $\omega(n)$ is even, that is, those $n$ with an even number of distinct prime factors.

Corollary 2.7. If $x \geqq 2$, then

$$
B_{\alpha}(x, E)=\left(\frac{3 \zeta(2 \alpha+2) C_{\alpha+1}}{\pi^{2}(\alpha+1)}\right) x^{\alpha+1}+ \begin{cases}O\left(x^{\alpha+1 / 2}\right) & \text { if } \alpha>0 \\ O(\sqrt{ } x \log x) & \text { if } \alpha=0\end{cases}
$$

where

$$
C_{t}=1+\prod_{p}\left(1-\frac{2}{p^{2 t}}\right), \quad t>\frac{1}{2} .
$$

Proof. It remains to evaluate $\zeta_{E}(s), s>1$. We have

$$
\begin{aligned}
\zeta_{E}(s) & =\sum_{n=1}^{\infty} \frac{1}{\sum_{\omega(n) \text { even }}}=\frac{1}{2}\left\{\sum_{n=1}^{\infty} \frac{1}{n^{s}}+\sum_{n=1}^{\infty} \frac{(-1)^{\omega(n)}}{n^{s}}\right\} \\
& =\frac{1}{2}\left\{\zeta(s)+\prod_{p}\left(1-\sum_{i=1}^{\infty} p^{-i s}\right)\right\}=\frac{1}{2}\left\{\zeta(s)+\prod_{p}\left(2-\frac{1}{1-p^{-s}}\right)\right\} \\
& =\frac{1}{2}\left\{\zeta(s)+\prod_{p}\left(\frac{1}{1-p^{-s}}\right) \cdot \prod_{p}\left(1-2 p^{-s}\right)\right\}=\frac{\zeta(s)}{2}\left\{1+\prod_{p}\left(1-\frac{2}{p^{s}}\right)\right\} .
\end{aligned}
$$

This proves (2.22).

Corollary $2.7 .1(\alpha=0)$. If $x \geqq 2$, then

$$
B(x, E)=\frac{C x}{2}+O(\sqrt{ } x \log x),
$$

where $C=C_{1}$, as defined by (2.23).

Corollary 2.7.2 (Feller and Tornier [10, §12]; also CF. Schoenberg $[15, \S 10])$. The asymptotic density of the sequence of $n$ for which $\omega(Q(n))$ is even is

$$
\frac{1}{2}\left(1+\prod_{p}\left(1-\frac{2}{p^{2}}\right)\right) .
$$

Finally, we consider the case in which $S$ is the set $F$ of those $n$ for which $\Omega(n)$ is even. One obtains in this case

COROLLARY 2.8. If $x \geqq 2$, then

$$
B_{\alpha}(x, F)=\left(\frac{3 \zeta(2 \alpha+2) c_{\alpha+1}}{\pi^{2}(\alpha+1)}\right) x^{\alpha+1}+ \begin{cases}O\left(x^{\alpha+1 / 2}\right) & \text { if } \alpha>0 \\ O(\sqrt{ } x \log x) & \text { if } \alpha=0\end{cases}
$$

where

$$
c_{t}=1+\frac{\zeta(4 t)}{\zeta^{2}(2 t)}, \quad t \geqq 1
$$


The proof is similar to that of Corollary 2.7.

Corollary $2.8 .1(\alpha=0)$. If $x \geqq 2$, then

$$
B(x, F)=\frac{7 x}{10}+O(\sqrt{ } x \log x) .
$$

Corollary 2.8.2 (CESÀro [2, §20]). The asymptotic density of the set of $n$ for which $\Omega(Q(n))$ is even is equal to $7 / 10$.

3. Problem II. Estimates for $B_{\alpha}^{\prime}(x, S)$. Corresponding to (2.1) we define

$$
\Gamma_{\alpha}^{\prime}(n, S)=\left\{\begin{array}{cl}
\left(\frac{n}{Q^{2}(n)}\right)^{\alpha} & \text { if } Q(n) \in S, Q(n) \| n, \\
0 & \text { otherwise. }
\end{array}\right.
$$

In case $\alpha=0, \Gamma_{\alpha}^{\prime}(n, S)$ reduces to the characteristic function of the sequence of $n$ for which $Q(n)$ is a unitary divisor of $n$ and $Q(n) \in S$. From (1.1) one obtains

LEMMA 3.1 .

$$
\Gamma_{\alpha}^{\prime}(n, S)=\sum_{d^{2} * e=n \cdot d \in S} e^{\alpha} \mu^{2}(e)
$$

We shall also need the following generalization of Lemma 2.2.

LEMMA 3.2 [4, LeMMA 4.2]. If $s \geqq 0$ and $r$ is a fixed positive integer, then

$$
\mho_{r, s}(x) \equiv \sum_{n \leqq x ;(n, r)=1} \mu^{2}(n) n^{s}=\frac{6 r}{\pi^{2} \psi(r)}\left(\frac{x^{s+1}}{s+1}\right)+O\left(\theta(r) x^{s+1 / 2}\right),
$$

where $\theta(r)$ denotes the number of square-free divisors of $r$ and $\psi(r)$ is Dedekind's $\psi$-function,

$$
\psi(r)=r \prod_{p \mid r}\left(1+\frac{1}{p}\right)=\sum_{d \delta=r} \mu^{2}(d) \delta .
$$

REMARK 3.1. Note that $r / \psi(r)=O(1)$.

THEOREM 3.1. If $\alpha \geqq 0$, then for $x \geqq 2$,

$$
B_{\alpha}^{\prime}(x, S)=\frac{6}{\pi^{2}}\left(\frac{x^{\alpha+1}}{\alpha+1}\right) \zeta_{S}^{\prime}(2 \alpha+1)+O\left(x^{\alpha+1 / 2} R_{\alpha}^{\prime}(x, S)\right)
$$

where

$$
\zeta_{S}^{\prime}(s)=\sum_{n=1 ; n \in S}^{\infty} \frac{1}{n^{s} \psi(n)}, \quad s>0
$$

and where

$$
R_{\alpha}^{\prime}(x, S)=\sum_{\sqrt{ } x \cdot n \in S} \frac{\theta(n)}{n^{2 \alpha+1}}
$$


unless the sum is vacuous, in which case $R_{\alpha}^{\prime}(x, S)=1$. The O-constant is independent of the set $S$.

REMARK 3.2. Since $\sum_{n \leqq x} \theta(n) / n=O\left(\log ^{2} x\right)$ (cf. $\left.[5,(3.4)]\right)$,

$$
R_{\alpha}^{\prime}(x, S)= \begin{cases}O(1) & \text { if } \alpha>0 \\ O\left(\log ^{2} x\right) & \text { if } \alpha=0\end{cases}
$$

In addition, if $\theta(n)$ is bounded on $S, R_{0}^{\prime}(x, S)=O(\log x)$, while, if $S$ is finite, $R_{0}^{\prime}(x, S)=O(1)$.

Proof. By the definition of $B_{\alpha}^{\prime}(x, S)$ and Lemma 2.1,

$$
B_{\alpha}^{\prime}(x, S)=\sum_{n \leqq x} \Gamma_{\alpha}^{\prime}(n, S)=\sum_{d^{2} * e \leqq x ; d \in S} e^{\alpha} \mu^{2}(e)=\sum_{n \leqq \sqrt{ } ; n \in S} \mho_{n, \alpha}\left(\frac{x}{n^{2}}\right),
$$

so that by Lemma 3.2 and Remark 3.1,

$$
\begin{aligned}
B_{\alpha}^{\prime}(x, S) & =\frac{6}{\pi^{2}}\left(\frac{x^{\alpha+1}}{\alpha+1}\right) \sum_{n \leqq \sqrt{ } ; n \in S} \frac{1}{n^{2 \alpha+1} \psi(n)}+O\left(x^{\alpha+1 / 2} R_{\alpha}^{\prime}(x, S)\right) \\
& =\frac{6}{\pi^{2}}\left(\frac{x^{\alpha+1}}{\alpha+1}\right) \zeta_{S}^{\prime}(2 \alpha+1)+O\left(x^{\alpha+1} \sum_{n>\sqrt{ } x} \frac{1}{n^{2 \alpha+2}}\right)+O\left(x^{\alpha+1 / 2} R_{\alpha}^{\prime}(x, S)\right),
\end{aligned}
$$

from which the theorem results.

It is easily verified that for $s>0, t>0$,

$$
\zeta_{J}^{\prime}(s) \equiv \sum_{n=1}^{\infty} \frac{1}{n^{s} \psi(n)}=\zeta(s+1) \beta_{s+1}, \quad \beta_{t} \equiv \prod_{p}\left(1-\frac{p^{-t}}{p+1}\right) .
$$

Corollary $3.1(S=J)$. If $x \geqq 2$, then

$$
B_{\alpha}^{\prime}(x, J)=\frac{6}{\pi^{2}}\left(\frac{x^{\alpha+1}}{\alpha+1}\right) \zeta(2 \alpha+2) \beta_{2 \alpha+2}+ \begin{cases}O\left(x^{\alpha+1 / 2}\right) & \text { if } \alpha>0 \\ O\left(\sqrt{ } x \log ^{2} x\right) & \text { if } \alpha=0\end{cases}
$$

Place $B^{\prime}(x, S)=B_{0}^{\prime}(x, S)$. Then

Corollary 3.1.1 $(\alpha=0)$.

$$
B^{\prime}(x, J)=\beta^{\prime} x+O\left(\sqrt{ } x \log ^{2} x\right) \quad\left(\beta^{\prime}=\beta_{2}\right) .
$$

Letting $J^{\prime}$ denote the set of integers $n$ whose greatest square divisor is unitary, one obtains

COROLLARY 3.1.2. The sequence $J^{\prime}$ has asymptotic density $\beta_{2}$.

Next we consider the set $S$ consisting of the single integer $r$. 
Corollary 3.2. For all $\alpha \geqq 0$,

$$
B_{\alpha}^{\prime}(x, r)=\frac{6}{\pi^{2}}\left(\frac{x^{\alpha+1}}{\alpha+1}\right) \frac{r^{-2 \alpha-1}}{\psi(r)}+O\left(\frac{x^{1 / 2+\alpha} \theta(r)}{r^{2 \alpha+1}}\right),
$$

uniformly in $r \leqq \sqrt{ } x$.

Corollary 3.2.1 $(\alpha=0)$. The asymptotic density of the subset of $J^{\prime}$ consisting of those $n$ for which $Q(n)=r$ is $6 / r \psi(r) \pi^{2}$.

Let $S_{k}$ denote the set of $k$ th powers of the primes, $k \geqq 1, k$ fixed. Then

COROllary 3.2.2. For $x \geqq 3$,

$$
B^{\prime}\left(x, S_{k}\right)=\frac{6 x}{\pi^{2}}\left(\sum_{p} \frac{1}{p^{2 k-1}(p+1)}\right)+ \begin{cases}O(\sqrt{ } x) & \text { if } k>1 \\ O(\sqrt{ } x \log \log x) & \text { if } k=1\end{cases}
$$

Proof. This is a consequence of the theorem, in connection with (2.17).

COROLlary 3.2.3. The asymptotic density of the set of integers all of whose prime divisors are simple, with the exception of a single divisor of multiplicity $2 k$, is

$$
\frac{6}{\pi^{2}} \sum_{p} \frac{1}{p^{2 k-1}(p+1)}
$$

The case $k=1$ yields

COROllaRY 3.2.4 (RÉNYI [13, (6)]; AlSO CF. [7, (1.1)]). The set of integers whose prime divisors are all simple, with the exception of a single double divisor, has asymptotic density,

$$
\frac{6}{\pi^{2}} \sum_{p} \frac{1}{p(p+1)}
$$

Let $N_{s}(a)$ be defined as in $\S 2$.

COROLlaRY 3.3. The asymptotic density of the integers $n$ of $J^{\prime}$ for which $Q(n) \in N_{s}(a)$, is determined by

$$
\delta_{s}^{\prime}(a)=\frac{6}{\pi^{2}} \sum_{p_{1}, \ldots, p_{s}} \frac{1}{p_{1}^{2 a_{1}-1}\left(p_{1}+1\right) \cdots p_{s}^{2 a_{s}-1}\left(p_{s}+1\right)},
$$

where the summation satisfies the conditions described in Corollary 2.6.1.

Note that the case $s=1$ of this result yields a second proof o 1Corollary 3.2.3.

Let now $d_{m}^{\prime}$ have the same significance as in the introduction. By Corollary 3.3, one finds that 
COROllary 3.3.1. If $|z|<2$,

$$
\sum_{m=0}^{\infty} d_{m}^{\prime} z^{m}=\frac{6}{\pi^{2}} \prod_{p}\left(1+\frac{p z}{(p+1)\left(p^{2}-z^{2}\right)}\right) .
$$

The proof is analogous to that of Corollary 2.6.2 and is therefore omitted.

Let $E$ denote the set of $n$ with $\omega(n)$ even.

COROLlaRY 3.4. The asymptotic density of the set of $n$ contained in $J^{\prime}$, such that $\omega(Q(n))$ is even, is given by

$$
\frac{1}{2}\left(\beta^{\prime}+\gamma^{\prime}\right), \quad \beta^{\prime}=\beta_{2}, \quad \gamma^{\prime}=\prod_{p}\left(1-\frac{2 p+1}{p^{2}(p+1)}\right),
$$

where $\beta_{t}$ is defined by (3.10).

The proof is similar to that of Corollary 2.7.2.

As in $\S 2$, let $F$ denote the sequence of $n$ such that $\Omega(n)$ is even. Analogous to Corollary 3.4 , one may prove

COROLlaRY 3.5. The asymptotic density of the sequence of $n$ for which $n \in J^{\prime}$ and $\Omega(Q(n))$ is even is $\left(5 \beta^{\prime}+2 \delta^{\prime}\right) / 10$, where

$$
\beta^{\prime}=\prod_{p}\left(1-\frac{1}{p^{2}(p+1)}\right), \quad \delta^{\prime}=\prod_{p}\left(1+\frac{1}{p^{2}(p+1)}\right) .
$$

REMARK 3.3. Corollary 3.1 .2 also results from the case $z=1$ in (1.9), or equivalently (3.16), by virtue of (1.10) in connection with the following principle: Let $G_{1}, G_{2}, \cdots$ be mutually disjoint sets of integers with densities $D\left(G_{i}\right), i \geqq 1$, such that $J=\bigcup_{i=1}^{\infty} G_{i}$ and $\sum_{1}^{\infty} D\left(G_{i}\right)=1$; if $J^{*} \subseteq J$ and $\Gamma=\bigcup_{i \in J^{*}} G_{i}$, then $D(\Gamma)$ exists and

$$
D(\Gamma)=\sum_{i \in J^{*}} D\left(G_{i}\right)
$$

4. Problem III. Estimates for $B_{\alpha}^{*}(x, T)$. Let $L$ have the meaning of $\S 1$ and suppose that $T$ is a nonvacuous subset of $L$. We define

$$
\Gamma_{\alpha}^{*}(n, T)=\left\{\begin{array}{cl}
\left(\frac{n}{Q^{*}(n)}\right)^{\alpha} & \text { if } Q^{*}(n) \in T, \\
0 & \text { otherwise. }
\end{array}\right.
$$

Evidently, $\Gamma_{0}^{*}(n, T)$ is the characteristic function of the sequence of $n$ for which $Q^{*}(n) \in T$. The uniqueness of the factorization (1.4) leads to

LEMMA 4.1.

$$
\Gamma_{\alpha}^{*}(n, T)=\sum_{d * e=n ; d \in T} e^{\alpha} \mu^{2}(e)
$$


Let $L(x)$ denote the enumerative function of $L$, representing the number of $n \leqq x$ contained in $L$. It is an elementary fact (cf. $[8, \S 1])$ that

$$
R(x)=O(\sqrt{ } x)
$$

We now prove

THEOREM 4.1. If $\alpha \geqq 0$, then for $x \geqq 2$,

$$
B_{\alpha}^{*}(x, T)=\frac{6}{\pi^{2}}\left(\frac{x^{\alpha+1}}{\alpha+1}\right) \zeta_{T}^{*}(\alpha)+O\left(x^{\alpha+1 / 2} R_{\alpha}^{*}(x, T)\right),
$$

where $\zeta_{T}^{*}(\alpha)=\zeta_{T}^{\prime}(\alpha)$, as defined by (3.6), and

$$
R_{\alpha}^{*}(x, T)=\sum_{n \leqq x ; n \in T} \frac{\theta(n)}{n^{\alpha+1 / 2}},
$$

if this sum is nonvacuous; otherwise, $R_{\alpha}^{*}(x, T)=1$. The O-constant in (4.4) is not dependent upon the set $T$; moreover, Remark 3.2 is valid here if $S$ is replaced by $T$ and $R_{\alpha}^{\prime}(x, S)$ by $R_{\alpha}^{*}(x, T)$.

Proof. By Lemma 3.1

$$
B_{\alpha}^{*}(x, T)=\sum_{n \leqq x} \Gamma_{\alpha}^{*}(n, T)=\sum_{d e \leqq x ; d \in T ;(d, e)=1} e^{\alpha} \mu^{2}(e)=\sum_{n \leqq x ; n \in T} \mho_{n, \alpha}\left(\frac{x}{n}\right),
$$

where $\mho_{r, s}$ is defined as in Lemma 3.2. Application of (3.3) gives

$$
\begin{aligned}
B_{\alpha}^{*}(x, T) & =\frac{6}{\pi^{2}}\left(\frac{x^{\alpha+1}}{\alpha+1}\right) \sum_{n \leqq \sqrt{ } x ; n \in T} \frac{1}{n^{\alpha} \psi(n)}+O\left(x^{\alpha+1 / 2} R_{\alpha}^{*}(x, T)\right) \\
& =\frac{6}{\pi^{2}}\left(\frac{x^{\alpha+1}}{\alpha+1}\right) \zeta_{T}^{*}(\alpha)+O\left(x^{\alpha+1} \sum_{n>x ; n \in T} \frac{1}{n^{\alpha+1}}\right)+O\left(x^{\alpha+1 / 2} R_{\alpha}^{*}(x, T)\right) .
\end{aligned}
$$

That $\zeta_{T}^{*}(\alpha)$ is finite for $\alpha \geqq 0$ is evident from the identity [9]

$$
\sum_{n \in L} \frac{1}{\psi(n)}=\frac{\pi^{2}}{6}
$$

By partial summation and (4.3),

$$
\begin{aligned}
\sum_{n>x ; n \in T} \frac{1}{n^{\alpha+1}} & =O\left(\sum_{n>x ; n \in L} \frac{1}{n^{\alpha+1}}\right)=O\left(\sum_{n>x} \frac{L(n)}{n^{\alpha+2}}\right)+O\left(\frac{L(x)}{x^{\alpha+1}}\right) \\
& =\sum_{n>x} \frac{1}{n^{\alpha+3 / 2}}+O\left(\frac{1}{x^{\alpha+1 / 2} y}\right)=O\left(\frac{1}{x^{\alpha+1 / 2}}\right),
\end{aligned}
$$

and (4.4) results from (4.7).

The final statement of the theorem concerning $R_{\alpha}^{*}(x, T)$ is a consequence of the same type of argument carried out in detail in $[5, \S 3]$ and will not be reproduced here. 
Observe that $\Gamma_{\alpha}^{*}(n, L)$ is $\gamma_{1}^{\alpha}(n)$ where $\gamma_{1}(n)$ is the greatest unitary square-free divisor of $n$.

Corollary 4.1 (IN CASE $\alpha \geqq 1$, CF. [4, Corollary 4.1.3]). If $\alpha>0$, then

$$
B_{\alpha}^{*}(x, L) \equiv \sum_{n \leqq x} \gamma_{1}^{\alpha}(n)=\frac{6}{\pi^{2}}\left(\frac{x^{\alpha+1}}{\alpha+1}\right) \zeta(\alpha+1) k_{\alpha}+O\left(x^{\alpha+1 / 2}\right),
$$

where $k_{\alpha}$ is defined by

$$
k_{\alpha}=\prod_{p}\left(1-\frac{p^{\alpha+1}+p^{\alpha}-1}{(p+1) p^{2 \alpha+1}}\right) .
$$

The case $\alpha=1$ gives

Corollary 4.1.1 (Cohen [4, Corollary 4.1.4]).

$$
\sum_{n \leqq x} \gamma_{1}(n)=\frac{k_{1} x^{2}}{2}+O\left(x^{3 / 2}\right)
$$

Let $N=N_{s}(a)$ be defined as in $\S 2$ and place $B_{0}^{*}(x, T)=B^{*}(x, T)$.

COROLlARY 4.2. Let $a_{1}, \cdots, a_{s}$ be an s-tuple of integers, each $>1$. Then for $x \geqq 3$,

$$
B^{*}(x, N)=\left(\frac{6 x}{\pi^{2}}\right) \zeta_{N}^{*}(1)+O\left(\sqrt{ } x(\log \log x)^{t}\right)
$$

where $t$ is the number of 2's in the set $a_{1}, \cdots, a_{s}$.

Proof. By (2.17) and the fact that $\theta(n)$ is bounded on $N_{s}(a)$.

COROLlaRY 4.2.1 (RÉNYI $[13,(17)]$ ). The asymptotic density of the integers $n$ for which $Q^{*}(n) \in N_{s}(a)$, where $a_{1}, \cdots, a_{s}$ are all $>1$, is given by

$$
\frac{6}{\pi^{2}} \sum_{p_{1}, \ldots, p_{s}} \frac{1}{p_{1}^{\alpha_{1}-1}\left(p_{1}+1\right) \cdots p_{s}^{a_{s}-1}\left(p_{s}+1\right)},
$$

where the summation satisfies the conditions of Corollary 2.6.1.

Corollary 4.2.2 (RÉNYI [13, $\left.\left(3^{\prime}\right)\right]$ ).

$$
\sum_{m=0}^{\infty} d_{m}^{*} z^{m}=\frac{6}{\pi^{2}} \prod_{p}\left(1+\frac{z}{(p+1)(p-z)}\right) .
$$

The proof is similar to that of Corollary 2.6.2.

One may observe that $\omega(Q(n))=\omega\left(Q^{*}(n)\right)$; hence Theorem 3 yields no new result analogous to Corollary 2.7.2. The corresponding problem for the $\Omega$-function, however, has the following solution, 
COROLLARY 4.3. The set of integers $n$ for which $\Omega\left(Q^{*}(n)\right)$ is even has asymptotic density,

$$
\frac{1}{2}+\frac{1}{5} \prod_{p}\left(1+\frac{p^{2}+p+1}{p^{3}(p+1)}\right) .
$$

\section{BIBLIOGRAPHY}

1. W. F. Bodmer, The limiting frequencies of integers with a given partitional characteristic, J. Roy. Statist. Soc. Ser. A 21 (1959), 134-143.

2. Ernesto Cesàro, Le plus grand diviseur carré, Ann. Mat. Pura Appl. 13 (1885), 251-268.

3. Eckford Cohen, Arithmetical functions associated with arbitrary sets of integers, Acta Arith. 5 (1959), 407-415.

4. - On an elementary method in the asymptotic theory of numbers, Duke Math. J. 28 (1961), 183-192.

5. - Arithmetical notes. V. A divisibility property of the divisor function, Amer. J. Math. 83 (1961), 693-697.

6. - Arithmetical notes. VII. Some classes of even functions $(\bmod r)$, Collect. Math. (to appear).

7. - Arithmetical notes. VIII. An asymptotic formula of Rényi, Proc. Amer. Math. Soc. 13 (1962), 536-539.

8. - On the distribution of certain sequences of integers, Amer. Math. Monthly 70. (1963), 516-521.

9. - A property of Dedekind's $\psi$-function, Proc. Amer. Math. Soc. 12 (1961), 996.

10. W. Feller and E. Tornier, Mengentheoretische Untersuchungen von Eigenschaften der Zahlenreihe, Math. Ann. 107 (1933), 188-232.

11. M. Kac, A remark on the preceding paper by A. Rényi, Acad. Serbe Sci. Publ. Inst. Math. 8 (1955), 163-165.

12. Hans-Joachim Kanold, Uber die Dichte von gewissen Zahlenmengen, J. Reine Angew. Math. 193 (1954), 250-252.

13. Alfred Rényi, On the density of certain sequences of integers, Acad. Serbe Sci. Publ. Inst. Math. 8 (1955), 157-162.

14. Peter Scherk, Bemerkungen zu einer Arbeit von Herrn Kanold, J. Reine Angew. Math. 196 (1956), 133-136.

15. I. J. Schoenberg, On asymptotic distributions of arithmetical functions, Trans. Amer. Math. Soc. 39 (1936), 315-330.

16. —_ On two theorems of P. Erdös and A. Rényi, Illinois J. Math. 6 (1962), 53-57.

\section{UNIVERSITY OF TENNESSEE,}

KNOXVILLE, TENNESSEE 